\title{
Pearson's Chi-Square Test Modifications for Comparison of Unweighted and Weighted Histograms and Two Weighted Histograms
}

\author{
N.D. Gagunashvili* \\ University of Akureyri, Borgir, v/Nordurslód, IS-600 Akureyri, Iceland \\ E-mail: nikolai@unak.is
}

Two modifications of the $\chi^{2}$ test for comparing usual (unweighted) and weighted histograms and two weighted histograms are proposed. Numerical examples illustrate an application of the tests for the histograms with different statistics of events. Proposed tests can be used for the comparison of experimental data histograms against simulated data histograms and two simulated data histograms

XI International Workshop on Advanced Computing and Analysis Techniques in Physics Research April 23-27 2007

Amsterdam, the Netherlands

${ }^{*}$ Speaker. 


\section{Introduction}

A frequently used technique in data analysis is the comparison of histograms. First suggested by Pearson [1] the $\chi^{2}$ test of homogeneity is used widely for comparing usual (unweighted) histograms. The modified $\chi^{2}$ test for comparison of weighted and unweighted histograms recently was proposed in [2].

This paper develops the ideas presented in [2]. From this development, two new results are presented. First, the $\chi^{2}$ test for comparing weighted and unweighted histograms is improved so that it can be applied for histograms with lower minimal number of events in a bin than is recommended in [2]. And secondly, a new $\chi^{2}$ test is proposed for the comparison two weighted histograms.

The paper is organized as follows. In section 2 the usual $\chi^{2}$ test and its application for the comparison of usual unweighted histograms is discussed. Tests for the comparison of weighted and unweighted histograms and two weighted histograms are proposed in sections 3 and 4 respectively. In section 5 the tests are illustrated and verified by a numerical example and experiments.

\section{2. $\chi^{2}$ test for comparison two (unweighted) histograms}

Without limiting the general nature of the discussion, we consider two histograms with the same binning and the number of bins equal to $r$. Let us denote the number of events in the $i$ th bin in the first histogram as $n_{i}$ and as $m_{i}$ in the second one. The total number of events in the first histogram is equal to $N=\sum_{i=1}^{r} n_{i}$, and $M=\sum_{i=1}^{r} m_{i}$ in the second histogram.

The hypothesis of homogeneity [3] is that the two histograms represent random values with identical distributions. It is equivalent that there exist $r$ constants $p_{1}, \ldots, p_{r}$, such that $\sum_{i=1}^{r} p_{i}=1$, and the probability of belonging to the $i$ th bin for some measured value in both experiments is equal to $p_{i}$. The number of events in the $i$ th bin is a random variable with a distribution approximated by a Poisson probability distribution $e^{-N p_{i}}\left(N p_{i}\right)^{n_{i}} / n_{i}$ ! for the first histogram and with distribution $e^{-M p_{i}}\left(M p_{i}\right)^{m_{i}} / m_{i}$ ! for the second histogram. If the hypothesis of homogeneity is valid, then the maximum likelihood estimator of $p_{i}, i=1, \ldots, r$, is

$$
\hat{p}_{i}=\frac{n_{i}+m_{i}}{N+M},
$$

and then

$$
X^{2}=\sum_{i=1}^{r} \frac{\left(n_{i}-N \hat{p}_{i}\right)^{2}}{N \hat{p}_{i}}+\sum_{i=1}^{r} \frac{\left(m_{i}-M \hat{p}_{i}\right)^{2}}{M \hat{p}_{i}}=\frac{1}{M N} \sum_{i=1}^{r} \frac{\left(M n_{i}-N m_{i}\right)^{2}}{n_{i}+m_{i}}
$$

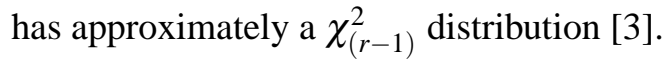

The comparison procedure can include an analysis of the residuals which is often helpful in identifying the bins of histograms responsible for a significant overall $X^{2}$ value. Most convenient for analysis are the adjusted (normalized) residuals [4]

$$
r_{i}=\frac{n_{i}-N \hat{p}_{i}}{\sqrt{N \hat{p}_{i}} \sqrt{(1-N /(N+M))\left(1-\left(n_{i}+m_{i}\right) /(N+M)\right)}} .
$$

If hypotheses of homogeneity are valid then residuals $r_{i}$ are approximately independent and identically distributed random variables having $\mathscr{N}(0,1)$ distribution. Notice that residuals (2.3) are 
related with the first histogram and residuals related with the second histogram are:

$$
r_{i}^{\prime}=\frac{m_{i}-M \hat{p}_{i}}{\sqrt{M \hat{p}_{i}} \sqrt{(1-M /(N+M))\left(1-\left(n_{i}+m_{i}\right) /(N+M)\right)}} .
$$

As $r_{i}=-r_{i}^{\prime}$, it makes sense either to use residuals (2.3) or (2.4).

The application of the $\chi^{2}$ test has restrictions related to the value of the expected frequencies $N p_{i}, M p_{i}, i=1, \ldots, r$. A conservative rule formulated in [何] is that all the expectations must be 1 or greater for both histograms. The authors point out that this rule is extremely conservative and in the majority of cases the $\chi^{2}$ test may be used for histograms with expectations in excess of 0.5 in the smallest bin. In practical cases when expected frequencies are not known the estimated expected frequencies $M \hat{p}_{i}, N \hat{p}_{i}, i=1, \ldots, r$ can be used.

\section{Unweighted and weighted histograms comparison}

A simple modification of the ideas described above can be used for the comparison of the usual (unweighted) and weighted histograms. Let us denote the number of events in the $i$ th bin in the unweighted histogram as $n_{i}$ and the common weight of events in the $i$ th bin of the weighted histogram as $w_{i}$. The total number of events in the unweighted histogram is equal to $N=\sum_{i=1}^{r} n_{i}$ and the total weight of events in the weighted histogram is equal to $W=\sum_{i=1}^{r} w_{i}$.

Let us formulate the hypothesis of identity of an unweighted histogram to a weighted histogram so that there exist $r$ constants $p_{1}, \ldots, p_{r}$, such that $\sum_{i=1}^{r} p_{i}=1$, and the probability of belonging to the $i$ th bin for some measured value is equal to $p_{i}$ for the unweighted histogram and expectation value of weight $w_{i}$ equal to $W p_{i}$ for the weighted histogram. The number of events in the $i$ th bin is a random variable with distribution approximated by the Poisson probability distribution $e^{-N p_{i}}\left(N p_{i}\right)^{n_{i}} / n_{i}$ ! for the unweighted histogram. The weight $w_{i}$ is a random variable with a distribution approximated by the normal probability distribution $\mathscr{N}\left(W p_{i}, \sigma_{i}^{2}\right)$, where $\sigma_{i}^{2}$ is the variance of the weight $w_{i}$. If we replace the variance $\sigma_{i}^{2}$ with estimate $s_{i}^{2}$ (sum of squares of weights of events in the $i$ th bin) and the hypothesis of identity is valid, then the maximum likelihood estimator of $p_{i}, i=1, \ldots, r$, is

$$
\hat{p}_{i}=\frac{W w_{i}-N s_{i}^{2}+\sqrt{\left(W w_{i}-N s_{i}^{2}\right)^{2}+4 W^{2} s_{i}^{2} n_{i}}}{2 W^{2}} .
$$

We may then use the test statistic

$$
X^{2}=\sum_{i=1}^{r} \frac{\left(n_{i}-N \hat{p}_{i}\right)^{2}}{N \hat{p}_{i}}+\sum_{i=1}^{r} \frac{\left(w_{i}-W \hat{p}_{i}\right)^{2}}{s_{i}^{2}}
$$

and it is plausible that this has approximately a $\chi_{(r-1)}^{2}$ distribution.

This test, as well as the original one [3], has a restriction on the expected frequencies. The expected frequencies recommended for the weighted histogram is more than 25 . The value of the minimal expected frequency can be decreased down to 10 for the case when the weights of the events are close to constant. In the case of a weighted histogram if the number of events is unknown, then we can apply this recommendation for the equivalent number of events as $n_{i}^{\text {equiv }}=w_{i}^{2} / s_{i}^{2}$. 
The minimal expected frequency for an unweighted histogram must be 1 . Notice that any usual (unweighted) histogram can be considered as a weighted histogram with events that have constant weights equal to 1 .

The variance $z_{i}^{2}$ of the difference between the weight $w_{i}$ and the estimated expectation value of the weight is approximately equal to:

$$
\begin{gathered}
z_{i}^{2}=\operatorname{Var}\left(w_{i}-W \hat{p}_{i}\right)=N \hat{p}_{i}\left(1-N \hat{p}_{i}\right)\left(\frac{W s_{i}^{2}}{\sqrt{\left(N s_{i}^{2}-w_{i} W\right)^{2}+4 W^{2} s_{i}^{2} n_{i}}}\right)^{2} \\
+\frac{s_{i}^{2}}{4}\left(1+\frac{N s_{i}^{2}-w_{i} W}{\sqrt{\left(N s_{i}^{2}-w_{i} W\right)^{2}+4 W^{2} s_{i}^{2} n_{i}}}\right)^{2}
\end{gathered}
$$

The residuals

$$
r_{i}=\frac{w_{i}-W \hat{p}_{i}}{z_{i}}
$$

have approximately a normal distribution with mean equal to 0 and standard deviation equal to 1 .

\section{Two weighted histograms comparison}

Let us denote the common weight of events of the $i$ th bin in the first histogram as $w_{1 i}$ and as $w_{2 i}$ in the second one. The total weight of events in the first histogram is equal to $W_{1}=\sum_{i=1}^{r} w_{1 i}$, and $W_{2}=\sum_{i=1}^{r} w_{2 i}$ in the second histogram.

Let us formulate the hypothesis of identity of weighted histograms so that there exist $r$ constants $p_{1}, \ldots, p_{r}$, such that $\sum_{i=1}^{r} p_{i}=1$, and also expectation value of weight $w_{1 i}$ equal to $W_{1} p_{i}$ and expectation value of weight $w_{2 i}$ equal to $W_{2} p_{i}$. Weights in both the histograms are random variables with distributions which can be approximated by a normal probability distribution $\mathscr{N}\left(W_{1} p_{i}, \sigma_{1 i}^{2}\right)$ for the first histogram and by a distribution $\mathscr{N}\left(W_{2} p_{i}, \sigma_{2 i}^{2}\right)$ for the second. Here $\sigma_{1 i}^{2}$ and $\sigma_{2 i}^{2}$ are the variances of $w_{1 i}$ and $w_{2 i}$ with estimators $s_{1 i}^{2}$ and $s_{2 i}^{2}$ respectively. If the hypothesis of identity is valid, then the maximum likelihood and Least Square Method estimator of $p_{i}, i=1, \ldots, r$, is

$$
\hat{p}_{i}=\frac{w_{1 i} W_{1} / s_{1 i}^{2}+w_{2 i} W_{2} / s_{2 i}^{2}}{W_{1}^{2} / s_{1 i}^{2}+W_{2}^{2} / s_{2 i}^{2}} .
$$

We may then use the test statistic

$$
X^{2}=\sum_{i=1}^{r} \frac{\left(w_{1 i}-W_{1} \hat{p}_{i}\right)^{2}}{s_{1 i}^{2}}+\sum_{i=1}^{r} \frac{\left(w_{2 i}-W_{2} \hat{p}_{i}\right)^{2}}{s_{2 i}^{2}}=\sum_{i=1}^{r} \frac{\left(W_{1} w_{2 i}-W_{2} w_{1 i}\right)^{2}}{W_{1}^{2} s_{2 i}^{2}+W_{2}^{2} s_{1 i}^{2}}
$$

and it is plausible that this has approximately a $\chi_{(r-1)}^{2}$ distribution. The normalized or studentised residuals [6]

$$
r_{i}=\frac{w_{1 i}-W_{1} \hat{p}_{i}}{s_{1 i} \sqrt{1-1 /\left(1+W_{2}^{2} s_{1 i}^{2} / W_{1}^{2} s_{2 i}^{2}\right)}}
$$

have approximately a normal distribution with mean equal to 0 and standard deviation 1 . A recommended minimal expected frequency is equal to 25 for the proposed test. 

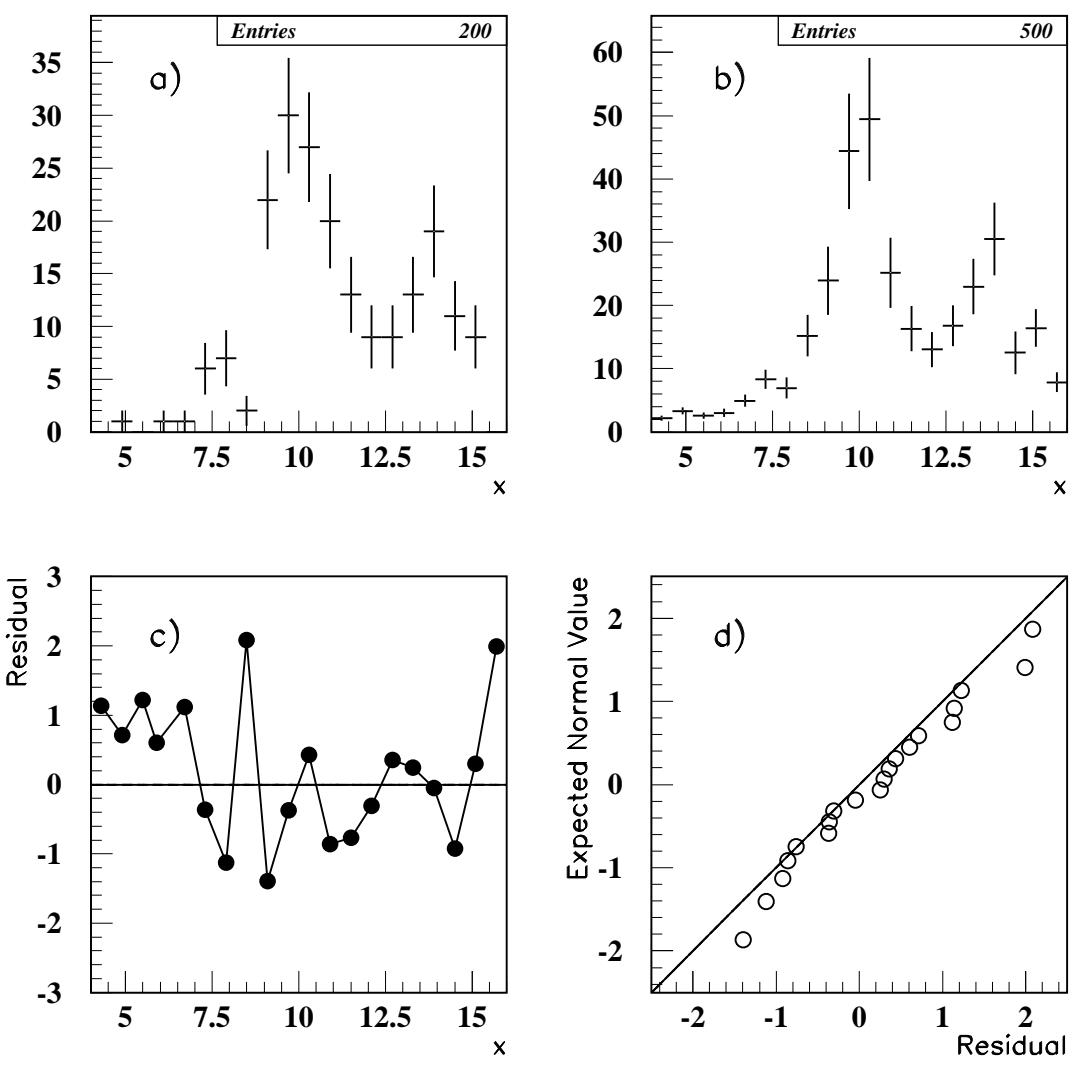

Figure 1: An example of comparison of the unweighted histogram with 200 events and the weighted histogram with 500 events: a) unweighted histogram; b) weighted histogram; c) normalized residuals plot; d) normal Q-Q plot of residuals.

\section{Numerical example and experiments}

The method described herein is now illustrated with an example. We take a distribution

$$
\phi(x)=\frac{2}{(x-10)^{2}+1}+\frac{1}{(x-14)^{2}+1}
$$

defined on the interval $[4,16]$. Events distributed according to the formula (5.1) are simulated to create the unweighted histogram. Uniformly distributed events are simulated for the weighted histogram with weights calculated by formula (5.1). Each histogram has the same number of bins: 20. Fig. 1 shows the result of comparison of the unweighted histogram with 200 events (minimal expected frequency equal to one) and the weighted histogram with 500 events (minimal expected frequency equal to 25)

The value of the test statistic $X^{2}$ is equal to 21.09 with $p$-value equal to 0.33 , therefore the hypothesis of identity of the two histograms can be accepted. The behavior of the normalized 


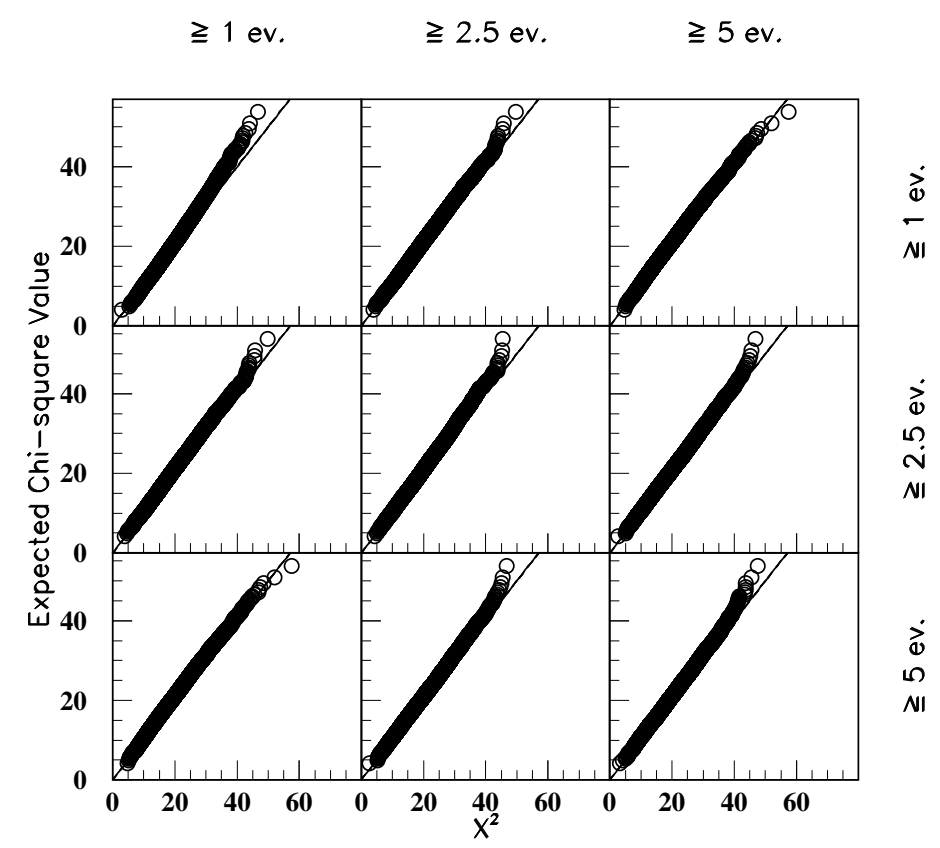

Figure 2: Chi-square Q-Q plots of $X^{2}$ statistics for two unweighted histograms with different minimal expected frequencies.

residuals plot (see Fig. 1c) and the normal Q-Q plot (see Fig. 1d) of residuals are regular and we cannot identify the outliers or bins with a big influence on $X^{2}$.

To investigate the dependence of the distribution of the test statistics from the number of events all three tests were considered.

The comparison of pairs of unweighted histograms with different minimal expected frequencies was considered (Pearson's chi square test). Unweighted histograms with minimal expected frequencies equal to one (200 events), 2.5 (500 events) and 5 (1000 events) where simulated.

Fig. 2 shows the Q-Q plots of $X^{2}$ statistics for different pairs of histograms. In each case 10000 pairs of histograms were simulated.

As we can see for all cases the real distributions of test statistics are close to the theoretical $\chi_{19}^{2}$ distribution.

The comparison of pairs of unweighted and weighted histograms with different minimal expected frequencies was considered using the test proposed in section 3 above. Unweighted histograms with minimal expected frequencies equal to one (200 events), 2.5 (500 events) and 5 (1000 events) where simulated. Furthermore weighted histograms with minimal expected frequencies equal to 10 (200 events), 25 (500 events) and 50 (1000 events) where simulated. Fig. 3 shows the Q-Q plots of $X^{2}$ statistics for different pairs of histograms. As we can see the real distribution 


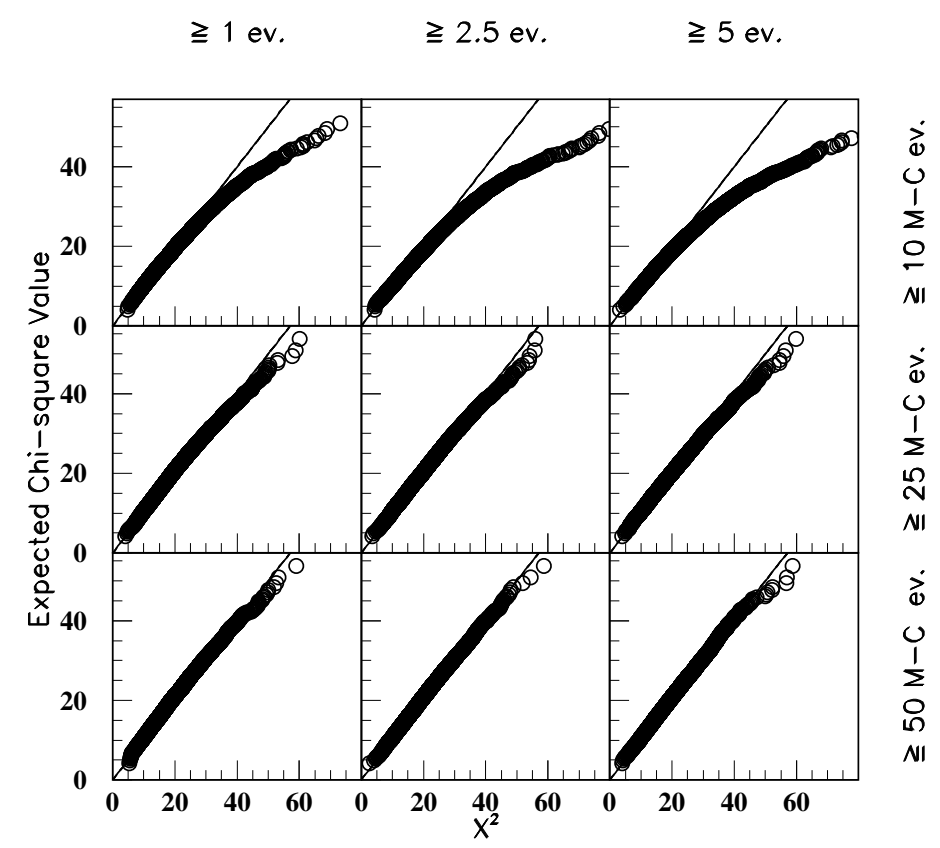

Figure 3: Chi-square Q-Q plots of $X^{2}$ statistics for unweighted and weighted histograms with different minimal expected frequencies.

of test statistics obtained for minimal expected frequency of weighted events, equal to 10 , has a heavier tail than the theoretical $\chi_{19}^{2}$ distribution. This means that the $p$-value calculated with the theoretical $\chi_{19}^{2}$ distribution is lower than the real $p$-value and any decision about the rejection of the hypothesis of identity of the two distributions is conservative. The distributions of test statistics for the minimal expected frequencies 25 and 50 are close to the theoretical distribution. This confirms that the minimal expected frequency 25 is reasonable restriction for the weighted histogram for this test.

The comparison of two weighted histograms with different minimal expected frequencies was considered using the test proposed in section 4 above. Weighted histograms with minimal expected frequencies equal to 10 (200 events), 25 (500 events) and 50 (1000 events) where simulated. Fig. 4 shows the Q-Q plots of $X^{2}$ statistics for different pairs of histograms. As we can see the real distributions of the test statistics are close to the theoretical $\chi_{19}^{2}$ distribution if the minimal expectations of the two histograms are close to each other, it is in all cases excluding case $(10,50)$. For the case when the difference in expectations are big $(10,50)$ the real distribution of the test statistics has a heavier tail than the theoretical $\chi_{19}^{2}$.

To verify the proposed tests two further numerical experiments were performed. For the first case unweighted histograms with minimal expected frequencies equal to 10 (2000 events), 25 (5000 


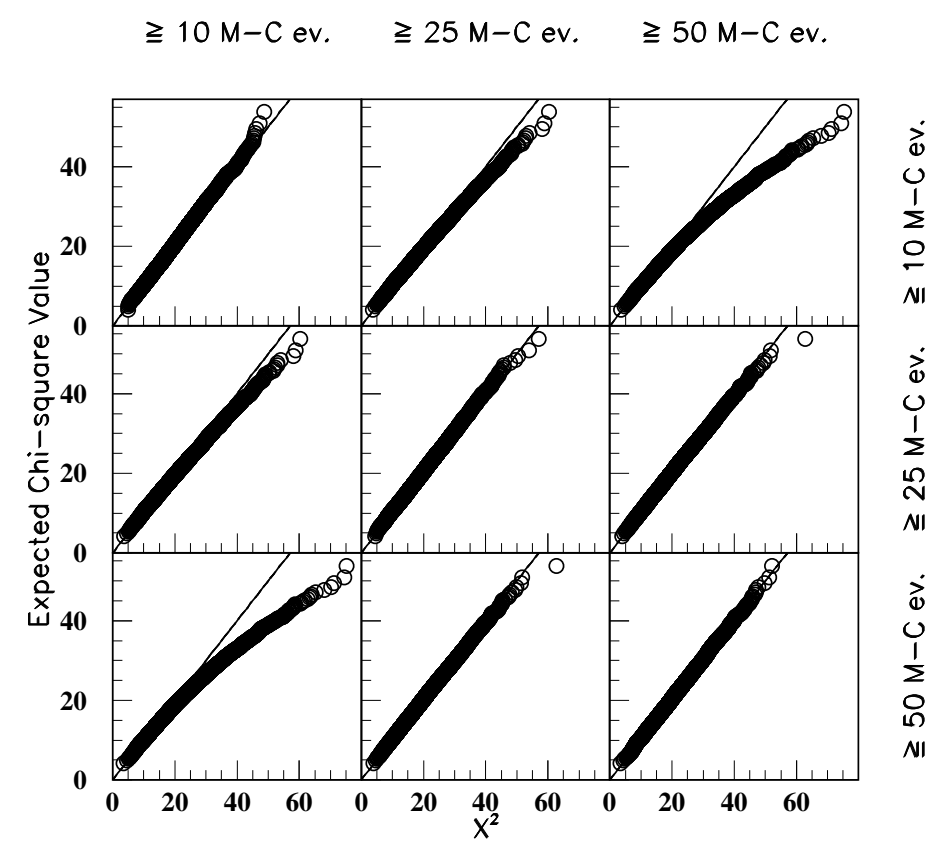

Figure 4: Chi-square Q-Q plots of $X^{2}$ statistics for two weighted histograms with different minimal expected frequencies.

events) and 50 (10000 events) were simulated. These histograms were compared to an unweighted histogram with 10 or more expected frequencies by the three methods described above. Fig. 5 shows the Q-Q plots of $X^{2}$ statistics for different pairs of histograms. As we can see the real distributions of the test statistics are close to the theoretical $\chi_{19}^{2}$ distribution for all three tests.

For the second case unweighted histograms with minimal expected frequencies equal to one (200 events), 2.5 (500 events) and 5 (1000 events) were simulated. These histograms were compared to an unweighted histogram with 10 or more expected frequencies by the first two methods described above. Fig. 6 shows the Q-Q plots of the $X^{2}$ statistics for different pairs of histograms. As we can see for all cases the real distributions of the test statistics are close to the theoretical $\chi_{19}^{2}$ distribution. Also the real distributions of the test statistics for the proposed method of comparison of unweighted and weighted histograms (see Fig. 6b) do not have heavy tails as is the case for a weighted histogram with weights calculated according formula (5.1) (see Fig. 3). This example confirms that the minimal expected frequency equal to 10 is enough for the application of the method of comparison of unweighted and weighted histograms if the weights of the events are close to a constant for the weighted histogram. 


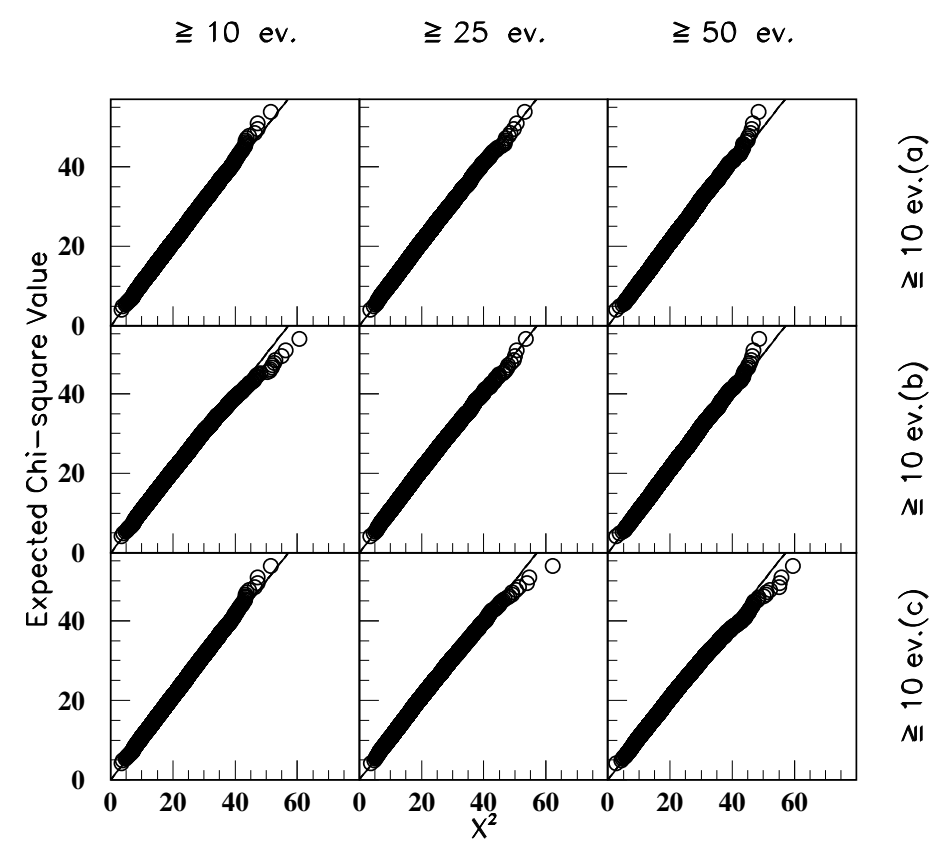

Figure 5: Chi-square Q-Q plots of $X^{2}$ statistics for two unweighted histograms with different tests: a) Pearson's chi square test; b) proposed in this article test for unweighted and weighted histograms; c) proposed in this article test for two weighted histograms.

\section{Conclusions}

A chi square test for comparing the usual (unweighted) histogram and the weighted histogram, together with a test for comparing two weighted histograms were proposed. In both cases formulas for normalized residuals were presented that can be useful for the identifications of bins that are outliers, or bins that have a big influence on $X^{2}$. For the first test the recommended minimal expected frequency of events is equal to 1 for an unweighted histogram and 25 for a weighted histogram. For the second test the recommended minimal expected frequency is equal to 25 . Numerical examples illustrated an application of the method for the histograms with different statistics of events and confirm that the proposed restrictions related with the expectations are reasonable. The developed approach can be generalized for a comparison of several unweighted and weighted histograms or just weighted histograms. The $X^{2}$ statistic has approximately a $\chi_{(r-1)(s-1)}^{2}$ distribution for $s$ histograms with $r$ bins.

The proposed in this paper tests are available in the ROOT framework under the class TH1:Chi2Test [叨] 


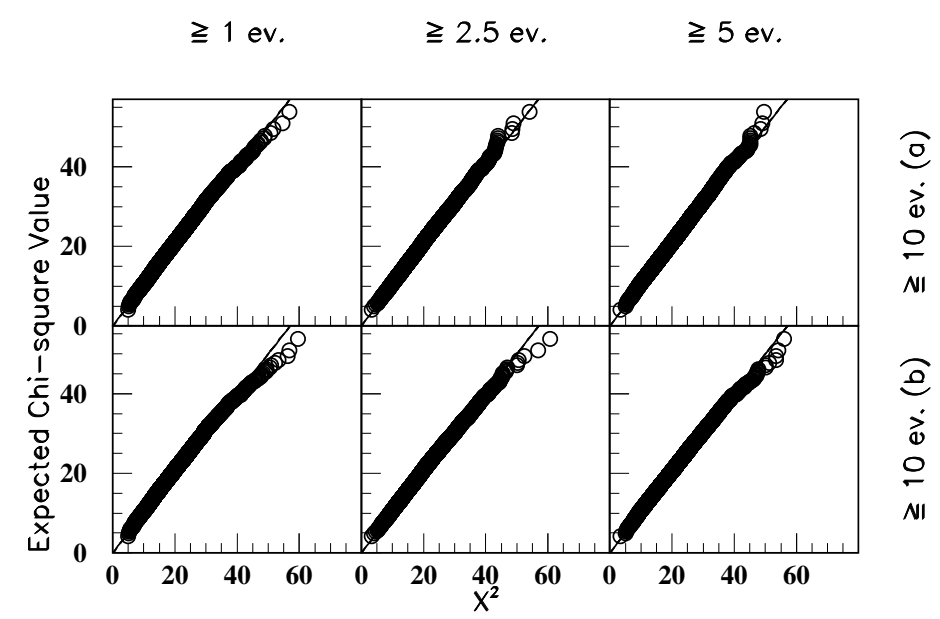

Figure 6: Chi-square Q-Q plots of $X^{2}$ statistics for two unweighted histograms with different tests: a) Pearson's chi square test; b) proposed in this article test for unweighted and weighted histograms.

\section{Acknowledgments}

The author is very grateful to Steffen Lauritzen (University of Oxford) who suggested idea of improving the method for comparing weighted and unweighted histograms, and to Mark O'Brien (University of Akureyri) for reading the paper in draft form and making constructive comments.

\section{References}

[1] K. Pearson, On the Theory of Contingency and Its Relation to Association and Normal Correlation, Drapers' Co. Memoirs, Biometric Series No. 1, London, 1904.

[2] N. Gagunashvili, Chi Square Test for the Comparison of Weighted and Unweighted Histograms, in proceedings of Conference on Statistical Problems in Particle Physics, Astrophysics and Cosmology, 12-15 September, 2005, Oxford , Imperial College Press, London, 2006.

[3] H. Cramer, Mathematical Methods of Statistics, Princeton University Press, Princeton, 1946.

[4] S. J. Haberman, The Analysis of Residuals in Cross-Classified Tables, Biometrics 29 (1973) 205.

[5] R. C. Lewontin and J. Felsenstein, The Robustness of Homogeneity Test in $2 x N$ Tables, Biometrics 21 (1965) 19.

[6] G. A. F. Seber, A. J. Lee, Linear Regression Analysis, John Wiley \& Sons Inc., New-York, 2003.

[7] http://root.cern.ch/root/htmldoc/TH1.html\#TH1:Chi2Test 